Review Article

\title{
Adjuvant Effects of Health Education of Chinese Medicine for Chronic Diseases: A Systematic Review and Meta-Analysis of Randomized Controlled Trials
}

\author{
An-Lu Wang, ${ }^{1,2}$ He Zhang, ${ }^{2}$ Jie Zhang, ${ }^{1}$ Yan Zhang, ${ }^{2}$ Hui-Juan Cao $\mathbb{D}^{1},{ }^{1}$ Jian-Ping Liu $\mathbb{D},{ }^{1}$ \\ Hao Xu $\left(\mathbb{D},{ }^{2}\right.$ and Ke-Ji Chen $\left(\mathbb{D}^{2}\right.$ \\ ${ }^{1}$ Beijing University of Chinese Medicine, Beijing, China \\ ${ }^{2}$ Xiyuan Hospital, China Academy of Chinese Medical Sciences, Beijing, China \\ Correspondence should be addressed to Hui-Juan Cao; huijuancao327@hotmail.com and Hao Xu; xuhaotcm@hotmail.com
}

Received 18 November 2019; Revised 17 January 2020; Accepted 21 February 2020; Published 31 March 2020

Academic Editor: Svein Haavik

Copyright (C) 2020 An-Lu Wang et al. This is an open access article distributed under the Creative Commons Attribution License, which permits unrestricted use, distribution, and reproduction in any medium, provided the original work is properly cited.

\begin{abstract}
Objective. To evaluate the adjuvant effects of health education of Chinese medicine (HECM) for patients with three types of common noncommunicable diseases (NCD-hypertension, diabetes, and coronary heart disease (CHD)). Methods. The protocol of this review was registered in the PROSPERO website (CRD42017058325). Six databases were searched till Sep. $30,2019$. Randomized controlled trials (RCTs) comparing HECM plus conventional therapy with conventional therapy were retrieved. Participants were diagnosed as one of the 3 above NCDs. HECM is regarded as lectures and classes about diet therapy, exercise therapy, emotion balance, and other knowledge according to Chinese medicine theory. The control rate of the disease was defined as a primary outcome in this review. Outcomes were synthesized using meta-analyses where reporting was sufficiently homogeneous or alternatively synthesized in a systematic review. Results. In total, 12 trials with 1142 patients were included in this review. Since all the trials may have unclear or high risk of bias, only low quality evidence could be found for supporting the adjunctive effect of HECM in treating hypertension, diabetes, and CHD, to reduce the control rate (risk ratio -1.58), the blood pressure level (mean difference $-9.38 \mathrm{mmHg}$ ), the fasting plasma glucose level (mean difference $-1.26 \mathrm{mmol} / \mathrm{L}$ ), and the symptoms of angina. Conclusion. The adjunctive effect of HECM on increasing the control rate of hypertension, improving the symptoms of diabetes and CHD, was only supported by low-quality evidence in this review. More rigorous trials with larger sample sizes and higher quality are warranted to provide a high quality of evidence.
\end{abstract}

\section{Introduction}

Noncommunicable diseases (NCDs) are chronic diseases of long duration and generally slow progression. In the MESH database (https://www.ncbi.nlm.nih.gov/mesh/), NCDs are defined as diseases which are typically noninfectious in origin and do not transmit from an affected individual to others. The four main types of NCDs are cardiovascular (e.g., heart attacks and stroke), cancer, chronic respiratory diseases (e.g., chronic obstructive pulmonary disease and asthma), and diabetes mellitus. It is reported that NCDs killed almost 40 million people annually, with nearly $70 \%$ of all deaths globally [1]. Cumulative economic losses of US $\$ 7$ trillion will be paid, and millions of people will be trapped in poverty over the next 15 years caused by the heavy burden from NCDs [2]. NCDs affect people in low- and middle-income countries more. In China, NCDs have become the leading causes of mortality and caused heavy economic burden as well. Cardiovascular diseases, hypertension, and diabetes mellitus caused millions of Chinese people's death each year [3, 4]. As we all know, with the changing of lifestyle and diets, the risk factors of NCDs are increased among the Chinese population, such as tobacco use, overuse of alcohol, physical inactivity, and unhealthy diet. Furthermore, metabolic risk factors, including raised blood pressure, overweight, hyperglycemia, and hyperlipidemia, increase the risk of NCDs. 
Therefore, besides the adequate treatments for lessening the impact of NCDs, it is important to focus on reducing the risk factors that cause NCDs. In the community population, the disease control of most NCDs patients (e.g., hypertension, diabetes mellitus, and coronary heart disease) relies on self-management, but the disease control rate (less than $50 \%$ for hypertension or diabetes) does not meet the expectations of health management departments. Thus, carrying out health education for community NCDs patients has become an attempted health service in China. Health education generally contains the education of rational drug usage, regular consultation, psychological supports, and lifestyle guidance. Evidence from systematic reviews showed that plenty of treatments of Chinese medicine, such as acupuncture, moxibustion, Tai Chi, and herbal medicine, may have clinical effects in treating specific NCDs [5-9]. Moreover, substantial trials suggested that health education of Chinese medicine (HECM) may be an effective complementary intervention when treating NCDs by controlling the risk factors of related diseases [10-15]. However, no systematic research evidence summarized the effectiveness of HECM. Therefore, we conduct this systematic review and meta-analysis to evaluate the adjuvant effects of HECM on the basis of conventional therapy in treating three common types of NCDs: hypertension, diabetes, and coronary heart disease (CHD).

\section{Methods}

The protocol of this review was registered in the PROSPERO website, which cited Wang Anlu, Cao Huijuan, and Liu Jianping. Chinese medicine health education for common noncommunicable diseases in community: a systematic review and meta-analysis of randomized controlled trials. PROSPERO 2017 CRD42017058325 is available at http:// www.crd.york.ac.uk/PROSPERO/display_record.php? ID $=$ CRD42017058325.

2.1. Inclusion Criteria. Eligible studies should be randomized controlled trials (RCTs) regardless of the language and publication status; the study setting should be located in the community and should meet the following criteria: (1) participants should be diagnosed as one of the three types of NCDs (hypertension, diabetes, or CHD) according to a recognized criterion. (2) Health education of Chinese medicine (HECM), such as lectures and classes about any life-style relevant knowledge based on Chinese medicine theory, as an adjunctive therapy of basic treatment (e.g., medications) for the specific NCDs is regarded as the intervention. The contents of HECM included diet education, mood balance education, exercise education, life style education according to Chinese medicine theory, and popularization of basic disease knowledge. Education was defined as a learning process in which doctors assisted patients in learning the contents of HECM instead of treating them directly. (3) Control should be the same basic treatment as in the HECM group; the basic treatment included standard treatment or conventional therapy for the specific NCDs. (4)
The primary outcome included the rehospitalization rate; all-cause mortality; control rate or key symptoms improvement (e.g., blood pressure for patients with hypertension, blood glucose and/or hemoglobin A1C (HbA1C) for patients with diabetes, and recurrence of cardiovascular events for patients with CHD). The secondary outcomes include other symptoms improvement, such as heart rate, cardiac function measured by echocardiogram, diabetes complication, blood lipid, patients' compliance of treatment, status of depression or anxiety measured by recognized scale (such as Hamilton Depression/Anxiety Scale), and patients' satisfactory for education/treatment.

2.2. Literature Search Strategy. We searched the following databases from their inception to 30 September 2019: PUBMED, Cochrane Central Register of controlled trials, Chinese Scientific Journal Database, China National Knowledge Infrastructure (CNKI), and Wanfang Database and Sino-Med Database. The searching strategy was used as follows:

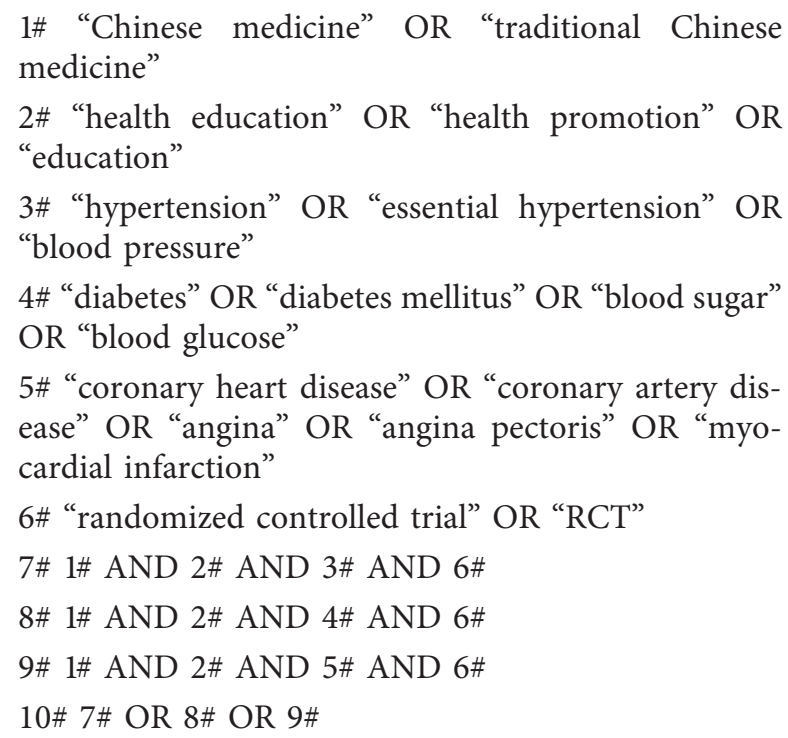

We also carefully scanned the references of all the eligible articles of RCTs to identify further publications.

2.3. Data Extraction and Quality Assessment. Retrieved studied were reviewed by two reviewers $(\mathrm{H}$. Zhang and $\mathrm{Y}$. Zhang) independently to screen the eligible trials according to the above criteria. Data of the included studies were then extracted based on a standard data-collection form, including first author name, publication year, regions, patient characteristics (sample size, gender, and age), methods of therapy, and outcomes. Differences between these two reviewers were discussed and resolved seriously. Another two reviewers (A. L. Wang and H. J. Cao) assessed the methodological quality of RCTs by using the Cochrane risk of bias tool (ROB) [16] independently. Disagreements were solved by consensus or consulting the third author (J. P. Liu). The ROB standard covers the following items: random sequence generation, allocation concealment, blinding of participants 
and personnel, blinding of outcome assessment, incomplete outcome data, selective reporting, and other bias. For each item, it can be assessed as low, high, or unclear risk of bias.

2.4. Statistical Analysis. RevMan 5.3 software provided by Cochrane Collaboration was used for data analyses. We calculated mean difference (MD) with corresponding 95\% confidence interval (CI) for continuous outcomes and risk ratio (RR) with its 95\% CI for dichotomous data. Quantitative meta-analysis was performed when the trials had similar characteristics regarding types of participants, intervention, comparison, outcomes measurements, and the statistical heterogeneity among trials was acceptable (the $I^{2}$ is less than 75\%). If necessary, we would conduct the subgroup analysis according to the age/gender of patients, the type of HECM, and the duration of the education. Publication bias would be assessed by funnel plot if data permitted.

2.5. Evidence Evaluation. The Grades of Recommendations Assessment, Development and Evaluation (GRADE) [17] was used to assess the quality of the evidence for each primary outcome with meta-analysis. Considering the following aspects, such as methodological quality, outcome consistency of trials, directness, and accuracy of evidence and possibility of publication bias, we judged whether to degrade the evidence of included trials and assessed the level of the evidence as high, moderate, low, or very low.

\section{Results}

3.1. Study Selection and Characteristics. The initial search retrieved 1122 articles from the six databases. After removing the duplicates, 618 trials were identified. Through screening the titles and abstracts, 60 trials remained. Moreover, 12 trials [18-29] with 13 articles [18-30] were included finally after reading the full-text (Figure 1).

The publication year of the included trials ranged from 2013 to 2019. All the trials were conducted in mainland China. In total, 1142 patients were included in these 12 trials. All trials were conducted in the community and more than $58.86 \%$ of participants were male. Six trials [18-23] focused on hypertensive patients, five trials [24-28] concerned diabetic patients, and one trial [29] included patients with coronary heart disease. Nine trials [19-25, 27, 29] reported the average duration of diseases, which range from 5 to 19 years.

All studies compared HECM plus routine western drugs to the drugs alone. HECM included lectures and classes about diet therapy, exercising training, emotion balance, massage guidance, manipulation (tuina) guidance and/or lavipeditum (zuyu) guidance based on Chinese medicine theory. Three articles $[20,21,24]$ reported the frequency of the education, which was once a week.

The hypertension-related outcomes included control rate [18, 21-23], level of blood pressure [19-23] (including systolic blood pressure (SBP) and diastolic blood pressure $(\mathrm{DBP}))$, disease awareness $[19,21]$, and compliance of treatment [21]. The diabetes-related outcomes included fasting plasma glucose (FPG) [24-28], HbA1c [25], disease awareness [25], and self-care activity [26, 28]. The CHDrelated outcomes included scores of Seattle angina questionnaire, symptoms of angina, and the consumption of nitroglycerin tablets [29] (details of the characteristics of the included trials are shown in Table 1).

3.2. Methodological Quality. Since the majority of included trials reported insufficient information to judge whether the method was likely to introduce bias, we assessed most of them as having an unclear risk of bias. Only five trials [24, 26-29] reported the methods of random sequence generation, which were assessed as having low risk of selection bias. However, none of them mentioned the allocation concealment methods. Performance bias is of high risk due to no study ever using placebo control or other methods to blind participants and personnel. Detection bias, attrition bias, reporting bias, and other biases were hard to determine due to the limited information provided in the reports. All trials reported neither dropout nor sample size calculation. Over all, the methodological quality of the included trials was not promising (Figure 2).

3.3. Effects of HECM. No trial reported the rehospitalization rate and all-cause mortality for any of the NCD.

\subsubsection{For Patients with Hypertension}

(1) Control Rate of Hypertension. Three trials [18, 22, 23] reported the control rate which counted by the proportion of number of patients whose blood pressure level back to normal (DBP less than $90 \mathrm{mmHg}$ ) or DBP decreased more than $20 \mathrm{mmHg}(\mathrm{RR}=1.58,95 \% \mathrm{CI} 1.21$ to $2.05, n=426$, $I^{2}=31 \%$ ); one trial [21] also reported the control rate of hypertension, which is defined as number of patients whose blood pressure level returned back to normal or DBP decreased more than $5 \mathrm{mmHg}(\mathrm{RR}=5.13,95 \%$ CI 2.68 to 9.80 , $n=100)$. We conducted subgroup meta-analysis for these four trials according to the definition of control (DBP decreased more than $20 \mathrm{mmHg}, 10 \mathrm{mmHg}$, or $5 \mathrm{mmHg}$ ); all subgroups showed better effect of HECM as adjunctive therapy for increasing the control rate of hypertension (Figure 3). However, there was obvious statistical heterogeneity among the subgroups $\left(I^{2}=90.9 \%\right)$.

Five trials [19-23] reported the level of SBP and DBP posttreatment. Meta-analysis showed significantly better effect of HECM combined with drugs for decreasing the level of SBP (MD $=-9.38 \mathrm{mmHg}, 95 \% \mathrm{CI}-10.51$ to $-8.25 \mathrm{mmHg}$, $n=560, I^{2}=75 \%$, Figure 4$)$ and DBP $(\mathrm{MD}=-6.38 \mathrm{mmHg}$, $95 \%$ CI -7.44 to $-5.32 \mathrm{mmHg}, I^{2}=29 \%, n=560$, Figure 5). Considering the obvious heterogeneity among trials, a subgroup meta-analysis was conducted according to patients' age for the level of SBP. It seems larger estimate effects are more likely to be found in elder patients (average age more than 75 years old) concerned about decreasing level of SBP. 


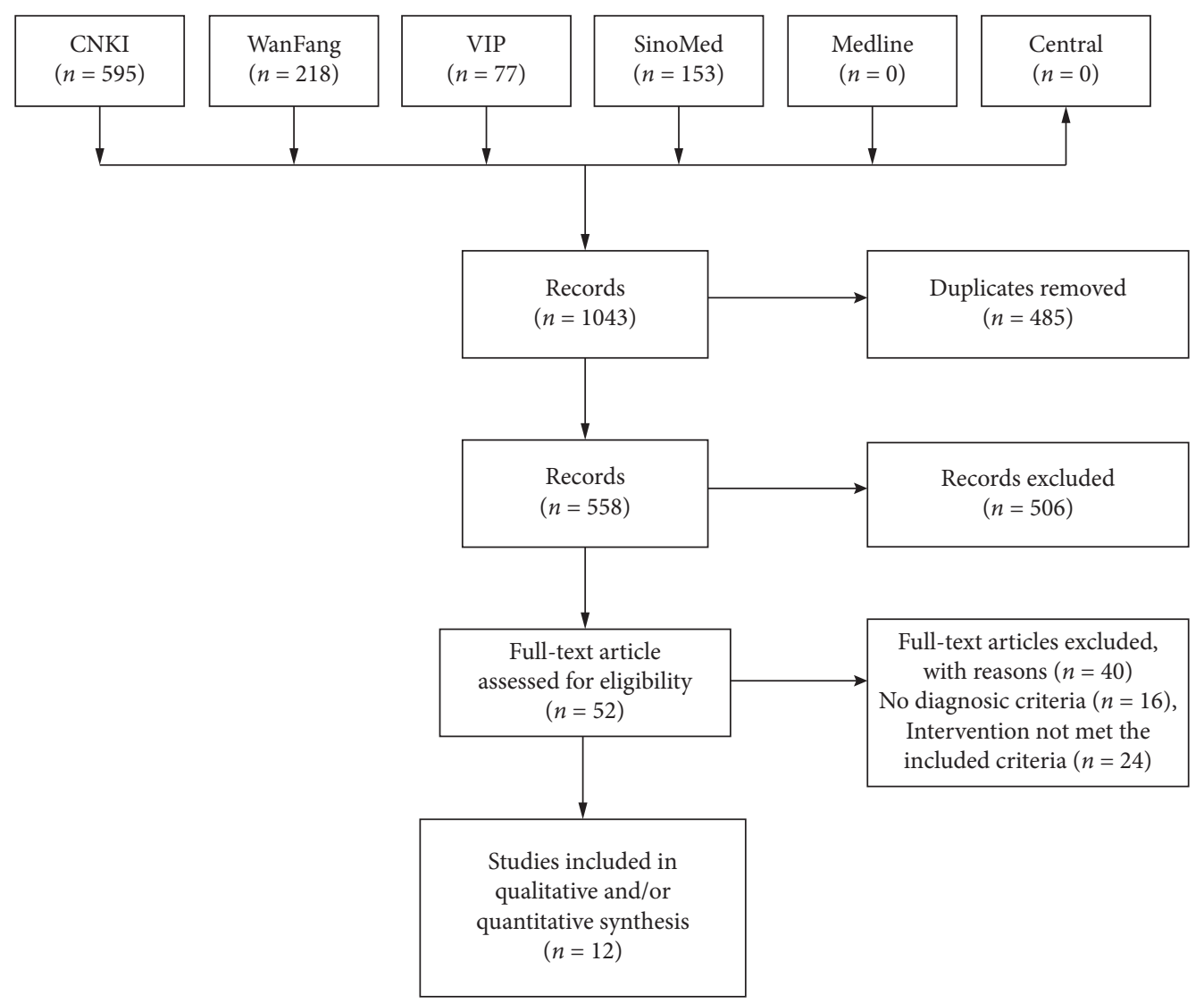

Figure 1: Study flow chart.

One trial [19] reported quality of life assessed by The Short Form Health Survey 36 (SF-36), which showed HECM group was superior to control group in four sections of SF36 , including physical functioning $(\mathrm{MD}=-2.53,95 \% \mathrm{CI}$ -4.15 to $-0.91, n=90)$, social role functioning $(\mathrm{MD}=0.57$, $95 \%$ CI 0.05 to $1.09, n=90)$, bodily pain ( $\mathrm{MD}=-1.17,95 \%$ CI -1.91 to $-0.43, n=90$ ), and emotional role functioning $(\mathrm{MD}=0.85,95 \%$ CI 0.48 to $1.22, n=90)$.

(2) Disease Awareness. Two trials [19, 21] reported hypertension-related questionnaire scores pre- and posttreatment. One trial [19] assessed patients knowledge for disease etiology and risk factors $(\mathrm{RR}=1.72,95 \% \mathrm{CI} 1.34$ to 2.20 , $n=90)$, clinical symptoms $(\mathrm{RR}=1.54,95 \%$ CI 1.24 , to 1.92 , $n=90)$, and treatment $(\mathrm{RR}=2.33,95 \%$ CI 1.66 to 3.27 , $n=90$ ) by counting the proportion of patients with good knowledge in those fields. The other trial [21] measured the patients' knowledge by scores of hypertension-related knowledge questionnaire $(\mathrm{MD}=52.90,95 \% \mathrm{CI} 47.79$ to 58.01, $n=100)$.

(3) Patients Compliance. One trial [21] reported patients' compliance to the medication and follow up, the result showed HECM may statistically have an effect on increasing the numbers of participants who adherence to the doctors' advice and regularly take drugs $(\mathrm{RR}=1.52,95 \% \mathrm{CI} 1.11$ to 2.09, $n=100)$.

\subsubsection{For Patients with Diabetes}

(1) FPG Level. Five trials [24-28] reported this outcome, which showed HECM combined with drugs was statistically more effective than drugs alone in decreasing the FPG level $(\mathrm{MD}=-1.26 \mathrm{mmol} / \mathrm{L}, 95 \% \mathrm{CI}-1.46$ to $-1.06 \mathrm{mmol} / \mathrm{L}$, $n=356, I^{2}=0 \%$, Figure 6).

The meta-analysis of 5 trials [24-28] showed good addon effect of HECM based on drugs $(\mathrm{MD}=-2.24 \mathrm{mmol} / \mathrm{L}$, $95 \% \mathrm{CI}-2.70$ to $-1.77 \mathrm{mmol} / \mathrm{L}, n=104, I^{2}=39 \%$, Figure 7 ) for controlling the $2 \mathrm{~h}$ postprandial plasma glucose.

(2) HbA1c Level. One trial [25] reported HbA1c level at the $6^{\text {th }}$ month, which showed that a combination group was statistically more effective than the control for this outcome $(\mathrm{MD}=-0.30 \%, 95 \% \mathrm{CI}-0.40$ to $-0.20 \%, n=104)$.

(3) Self-Control Activity. Two trials [26, 28] assessed the patients' self-control activity after intervention. One of them reported the proportion of patients who showed good selfmanagement behavior on appropriate exercise $(\mathrm{RR}=1.42$, 95\% CI 1.04 to $1.93, n=86)$, diet control ( $\mathrm{RR}=1.36,95 \% \mathrm{CI}$ 1.01 to $1.83, n=86)$, blood sugar monitor $(\mathrm{RR}=1.37,95 \% \mathrm{CI}$ 1.06 to $1.78, n=86)$, and regular medication $(\mathrm{RR}=1.31,95 \%$ CI 1.04 to $1.66, n=86$ ) between groups. Another one reported similar outcomes but were assessed by the reliability of the diabetes care profile (DCP). HECM combined with 


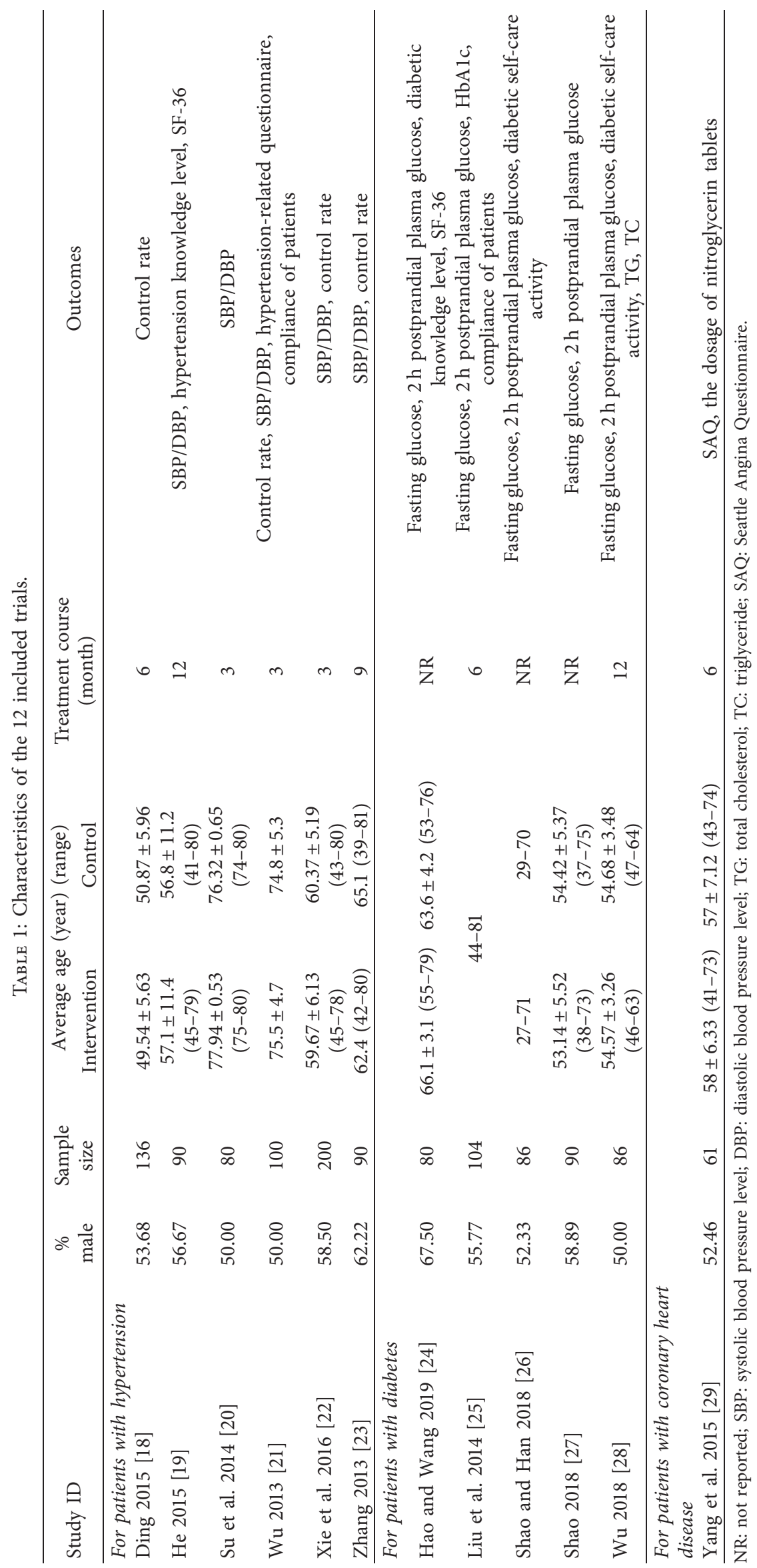




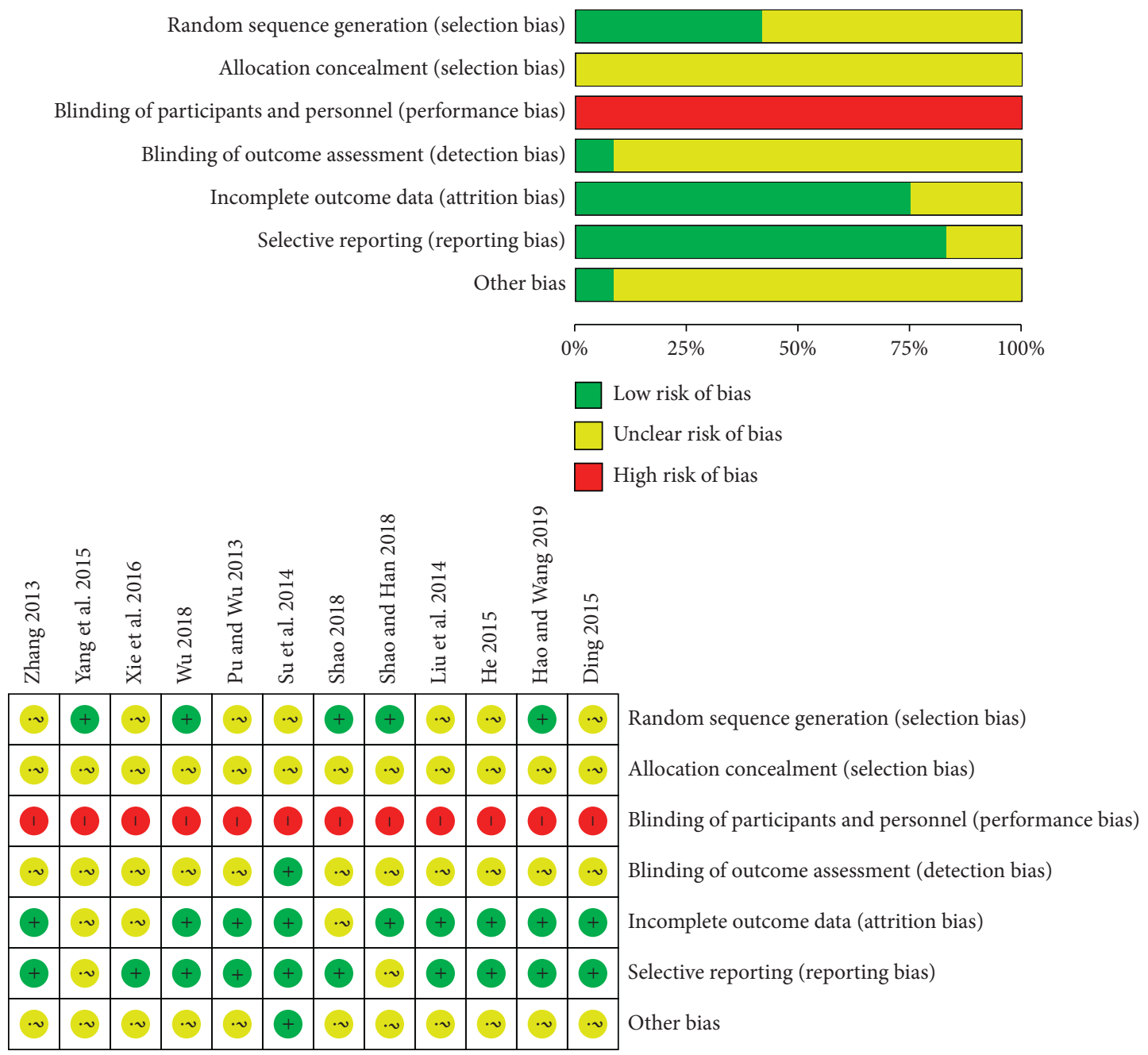

FIgURE 2: Risk of bias graph and summary of included studies.

drugs also showed better effect on increasing the patients self-control ability concerning appropriate exercise $(\mathrm{MD}=0.38,95 \%$ CI 0.26 to $0.50, n=86)$, diet control $(\mathrm{MD}=1.17,95 \% \mathrm{CI} 1.11$ to $1.23, n=86)$, blood sugar monitor $(\mathrm{MD}=0.94,95 \% \mathrm{CI} 0.73$ to $1.15, n=86)$, and regular medication $(\mathrm{MD}=1.23,95 \% \mathrm{CI} 1.12$ to $1.34, n=86)$.

(4) Patients Compliance. One trial [25] evaluated the patients' compliance according to the self-designed questionnaire. Of the total 50 scores, HECM may help in increasing the patients' compliance compared to the drugs group $(\mathrm{MD}=6.60,95 \% \mathrm{CI} 5.07$ to $8.13, n=104)$.

\subsubsection{For Patients with Coronary Heart Disease}

(1) Seattle Angina Questionnaire (SAQ). The only one study [29] (with 61 cases) concerning stable angina pectoris measured this outcome. Whether the total scores of SAQ $(\mathrm{MD}=4.90,95 \% \mathrm{CI} 0.57$ to 9.23$)$ or each of the five dimensions of coronary artery disease all showed the better effect of HECM in adjunctive with drugs. The five dimensions included physical limitation $(\mathrm{MD}=8.37,95 \% \mathrm{CI} 3.81$ to 12.93 ), angina stability ( $\mathrm{MD}=8.37,95 \% \mathrm{CI} 3.81$ to 12.93 ), angina frequency ( $\mathrm{MD}=3.93,95 \% \mathrm{CI} 0.98$ to 6.88$)$, treatment satisfaction ( $\mathrm{MD}=4.25,95 \% \mathrm{CI} 0.86$ to 7.64$)$, and disease perception $(\mathrm{MD}=7.74,95 \% \mathrm{CI} 4.50$ to 10.98$)$.

(2) The Dosage of Nitroglycerin Tablets. HECM group was superior to control on decreasing the monthly consumption of nitroglycerin tablets $(\mathrm{MD}=-5.52 \mathrm{mg}, 95 \% \mathrm{CI}-10.14$ to $-0.90 \mathrm{mg}, n=61)$.

3.4. Additional Analysis. Due to the limited number of included studies in one meta-analysis, the publication bias cannot be assessed by funnel plot. Meanwhile, no trials reported safety-related outcomes.

\section{Discussion}

4.1. Main Findings from the Review. Twelve trials with 1142 patients are included in this review. The meta-analysis showed a potential good adjunctive effect of HECM for the specific NCDs. For hypertension, HECM combined with drugs seemed to gain $21 \%$ more patients whose blood 


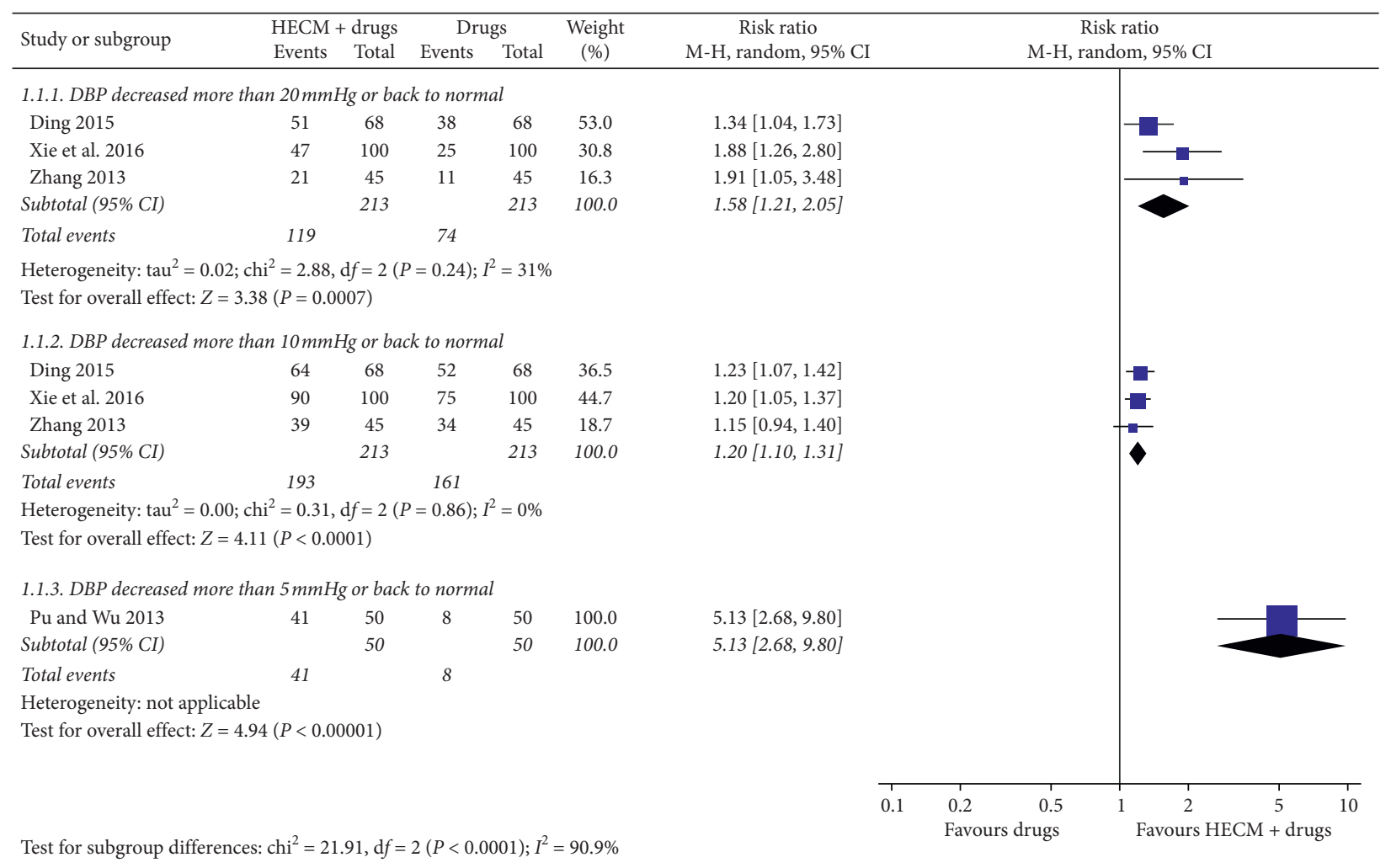

FIGURE 3: Forest plot of comparison of HECM plus drugs vs. drugs for hypertension on control rate SBP/DBP.

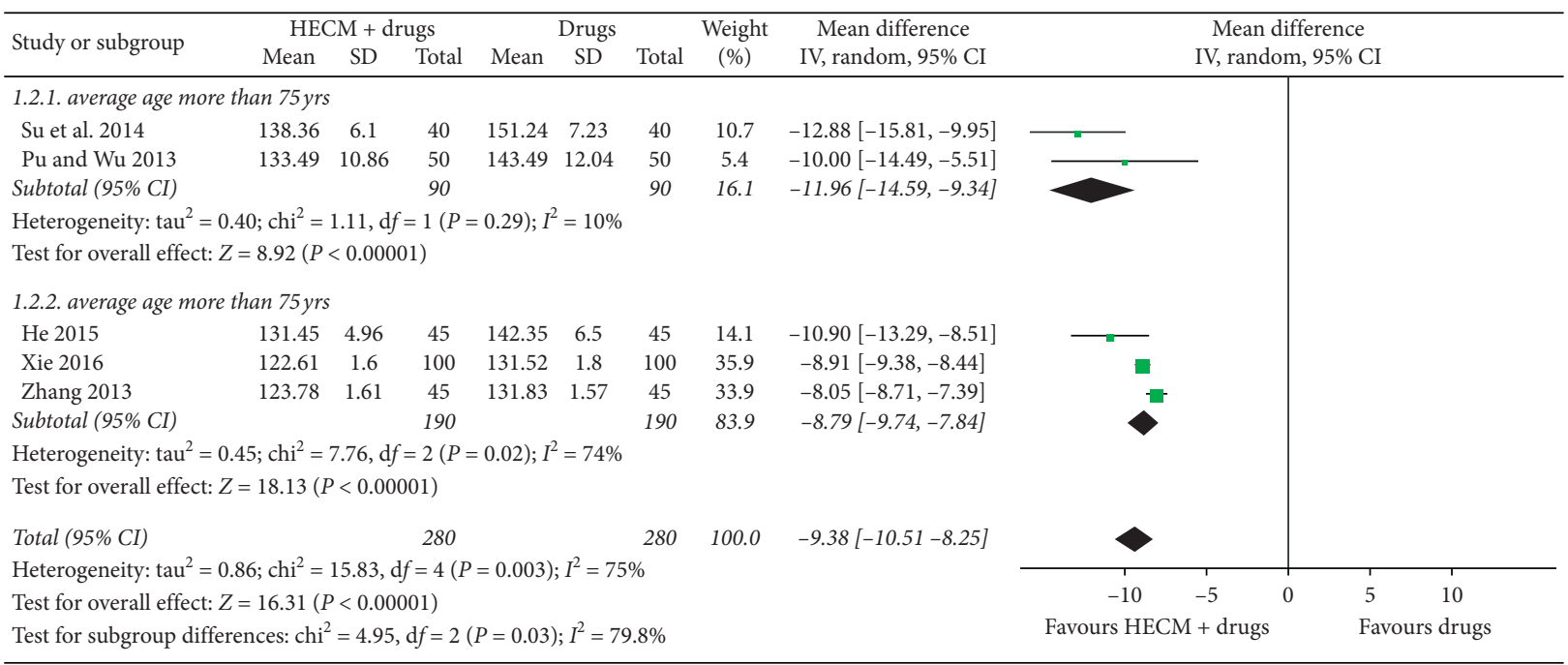

FIGURE 4: Forest plot of comparison of HECM plus drugs vs. drugs for hypertension on SBP.

pressure level was back to normal (lower than $90 \mathrm{mmHg}$ ). Meanwhile, combined with HCEM, drugs may decrease $9.38 \mathrm{mmHg}$ more SBP or $6.38 \mathrm{mmHg}$ more DBP after at least 3 months of treatment. For diabetes, HECM also was effective as an adjunctive therapy on controlling FPG level $(\mathrm{MD}=-1.26 \mathrm{mmol} / \mathrm{L}), 2 \mathrm{~h}$ postprandial plasma glucose $(\mathrm{MD}=-2.24 \mathrm{mmol} / \mathrm{L})$, and HbAlc level $(\mathrm{MD}=-30 \%)$ compared to drugs. For CHD, HECM seems to have more advantages in improving the symptoms of angina, to lower 4.9 scores of SAQ, and to reduce $5.52 \mathrm{mg}$ monthly consumption of nitroglycerin tablets. Though all the included trials did not mention any safety relevant outcomes, some of them reported that HECM may improve the patients' compliance and awareness of the disease.

4.2. Overall Quality of the Evidence. Due to the unclear or high risk of selection bias, performance bias, detection bias, and/or other bias for the majority of the included trials, the evidence for all outcomes should be downgraded for two 


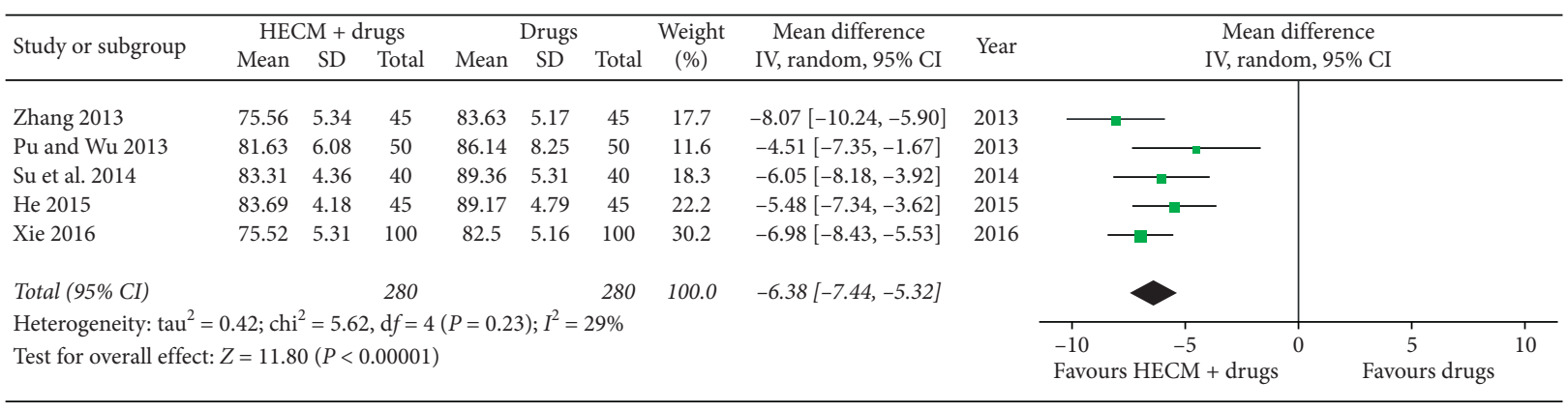

Figure 5: Forest plot of comparison of HECM plus drugs vs. drugs for hypertension on DBP quality of life.

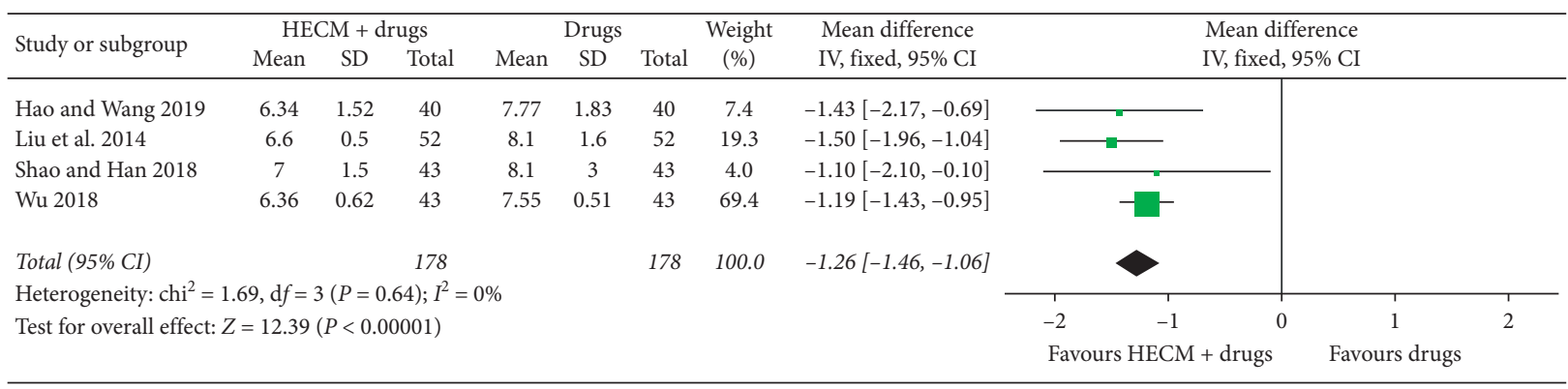

Figure 6: Forest plot of comparison of HECM plus drugs vs. drugs for diabetes on fast plasma glucose $2 \mathrm{~h}$ postprandial plasma glucose.

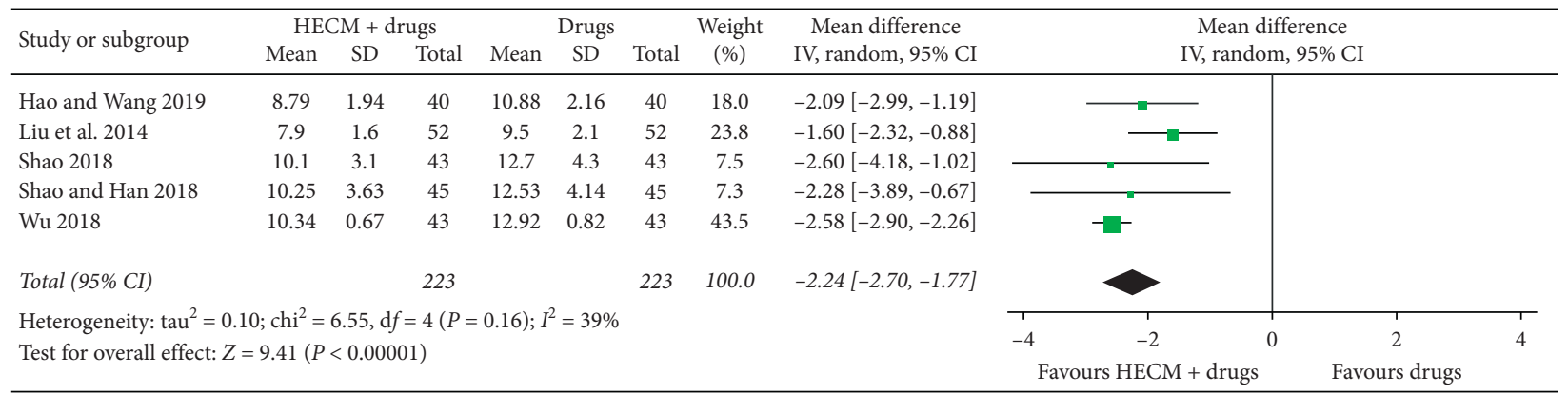

FIgURE 7: Forest plot of comparison of HECM plus drugs vs. drugs for diabetes on $2 \mathrm{~h}$ postprandial plasma glucose.

levels according to GRADE assessment. Besides, the sample size of the included trials was limited (less than 200 participants in meta-analysis with continuous outcomes or less than 300 events in meta-analysis with dichotomous outcomes), which lead to one level downgraded for imprecision. Publication bias was also suspected even though the funnel plot could not be done since all the studies were published in China with limited included participants, low quality, and all reported positive results to support the HECM application. Thus, only "very low" quality evidence could be provided to affirm the adjunctive effect of HECM for all the relevant outcomes in treating patients with hypertension, diabetes, or CHD. Summary of findings for the primary outcomes of the three specific NCDs is shown in Tables 2-4.

4.3. Reflections on the Effective Mechanism. The overall "very low" quality evidence showed adjunctive with HECM may increase $21 \%$ more patients whose blood pressure level was back to normal or at least decreased $20 \mathrm{mmHg}$ DBP after 3 months of medication treatment. It is very interesting when we looked through the results of the subgroup analysis shown in Figure 3. These four trials [19, 20, 22, 23] reported not only the control rate but also the numbers of patients whose DBP decreased at least $10 \mathrm{mmHg}$ in both groups, and the estimate value showed HECM group may have $15 \%$ more patients achieved this therapeutic effect. Compared to the first subgroup of control rate, it prompts that after 3 months of medication treatment, $75 \%$ of patients' DBP level may have more than $10 \mathrm{mmHg}$ reduction, but only $35 \%$ of patients' DBP may have more than $20 \mathrm{mmHg}$ reduction. However, for patients who were over 75 years old, only $16 \%$ of them may get more than $5 \mathrm{mmHg}$ reduction of DBP after medication treatment [21]. Subgroup analysis of posttreatment SBP level also found similar results that elder patients (over 75 years old) may achieve more SBP reduction $(\mathrm{MD}=-11.96 \mathrm{mmHg}, 95 \% \mathrm{CI}-14.59$ to $-9.34 \mathrm{mmHg})$ than younger patients $(\mathrm{MD}=-8.79 \mathrm{mmHg}, 95 \% \mathrm{CI}-9.74$ to $-7.84 \mathrm{mmHg}$ ) under HECM (see Figure 4). Despite the difference of drugs and other study application 
TABLe 2: Summary of findings for patients with hypertension.

HECM + drugs compared to drugs for diabetes

Patient or population: diabetes

Setting: community

Intervention: HECM + drugs

Comparison: drugs

Outcome

No. of participants (studies)

Control rate: DBP decreased more than

$20 \mathrm{mmHg}$ or back to normal assessed

with blood pressure level

No. of participants: 426 (3 RCTs)

Systolic blood pressure level (SBP)

No. of participants: 560 (5 RCTs)

\author{
Relative \\ effect $(95 \%$ \\ CI)
}

RR $1.58(1.21$

to 2.05$)$

The mean systolic blood
pressure level was
$\mathbf{1 3 5 . 9 1} \mathrm{mmHg}$
The mean diastolic blood
pressure level was
$\mathbf{8 5 . 8 6} \mathrm{mmHg}$

Anticipated absolute effects (95\% CI)

\begin{tabular}{|c|c|c|c|}
\hline Control & HECM $^{*}$ & Difference & Certainty \\
\hline $34.7 \%$ & $\begin{array}{l}54.9 \%(42 \\
\text { to } 71.2)\end{array}$ & $\begin{array}{l}20.2 \% \text { more ( } 7.3 \text { more to } \\
36.5 \text { more) }\end{array}$ & $\begin{array}{c}\oplus \bigcirc \bigcirc \bigcirc \\
\text { Very } \\
\text { low } \\
\text { a,b,c,d }\end{array}$ \\
\hline $\begin{array}{l}\text { an systolic blood } \\
\text { ure level was } \\
5.91 \mathrm{mmHg}\end{array}$ & - & $\begin{array}{l}\text { MD } 9.38 \mathrm{mmHg} \text { lower } \\
\text { (10.51 lower to } 8.25 \\
\text { lower) }\end{array}$ & $\begin{array}{l}\oplus \bigcirc \bigcirc \bigcirc \\
\text { Very } \\
\text { low }^{\text {a,b,d }}\end{array}$ \\
\hline $\begin{array}{l}\text { n diastolic blood } \\
\text { ure level was } \\
.86 \mathrm{mmHg}\end{array}$ & - & $\begin{array}{c}\text { MD } 6.38 \mathrm{mmHg} \text { lower } \\
(7.44 \text { lower to } 5.32 \\
\text { lower })\end{array}$ & $\begin{array}{l}\oplus \bigcirc \bigcirc \bigcirc \\
\text { Very } \\
\text { low }^{\text {a,d }}\end{array}$ \\
\hline
\end{tabular}

Diastolic blood pressure level (DBP)

No. of participants: 560 (5 RCTs)

GRADE working group grades of evidence.

High certainty: we are very confident that the true effect lies close to that of the estimate of the effect.

Moderate certainty: we are moderately confident in the effect estimate. The true effect is likely to be close to the estimate of the effect, but there is a possibility that it is substantially different.

Low certainty: our confidence in the effect estimate is limited. The true effect may be substantially different from the estimate of the effect.

Very low certainty: we have very little confidence in the effect estimate. The true effect is likely to be substantially different from the estimate of effect.

Explanations

(a) Most of the trials had an unclear risk of selection bias, detective bias, or other bias, all of them had a high risk of performance bias.

(b) There was potential statistical heterogeneity among trials ( $I$-square value $>50 \%$ ).

(c) Number of events less than 300 .

(d) All the included trials published in China with positive results and a small sample size.

${ }^{*}$ The risk in the intervention group (and its $95 \%$ confidence interval) is based on the assumed risk in the comparison group and the relative effect of the intervention (and its 95\% CI). CI: confidence interval; RR: risk ratio; MD: mean difference.

TABLE 3: Summary of findings for patients with diabetes.

HECM + drugs compared to drugs for diabetes

Patient or population: diabetes

Setting: community

Intervention: HECM + drugs

Comparison: drugs

Outcome

No. of participants

(studies)

Relative effect

(95\% CI)

Fast glucose (FPG)

No. of participants:

356 (4 RCTs)

$2 \mathrm{~h}$ postprandial plasma

glucose

No. of participants:

446 (5 RCTs)

GRADE working group grades of evidence

High certainty: we are very confident that the true effect lies close to that of the estimate of the effect.

Moderate certainty: we are moderately confident in the effect estimate. The true effect is likely to be close to the estimate of the effect, but there is a possibility that it is substantially different.

Low certainty: our confidence in the effect estimate is limited. The true effect may be substantially different from the estimate of the effect. Very low certainty: we have very little confidence in the effect estimate. The true effect is likely to be substantially different from the estimate of effect.

Explanations

(a) Most of the trials had an unclear risk of selection bias, detective bias, and other bias, all of them had a high risk of performance bias.

(b) All of the trials published in China with positive results and small sample size.

(c) There was potential statistical heterogeneity among trials ( $I$-square value more than $40 \%$ ).

* The risk in the intervention group (and its 95\% confidence interval) is based on the assumed risk in the comparison group and the relative effect of the intervention (and its 95\% CI). CI: confidence interval; MD: mean difference. 
TABLE 4: Summary of findings for patients with coronary heart disease.

HECM plus drugs compared to drugs for stable angina

Patient or population: stable angina

Setting: community

Intervention: HECM plus drugs

Comparison: drugs

Outcome

No. of participants

(studies)

Relative effect (95\% CI)

Seattle Angina

Questionnaire (SAQ)

No. of participants: 61 (1

RCT)

Nitroglycerin Tablets

consumption

No. of participants: 61

RCT)

GRADE working group grades of evidence

High certainty: we are very confident that the true effect lies close to that of the estimate of the effect.

Moderate certainty: we are moderately confident in the effect estimate. The true effect is likely to be close to the estimate of the effect, but there is a possibility that it is substantially different.

Low certainty: our confidence in the effect estimate is limited. The true effect may be substantially different from the estimate of the effect. Very low certainty: we have very little confidence in the effect estimate. The true effect is likely to be substantially different from the estimate of effect.

Explanations

(a) The trial had an unclear risk of selection bias, detective bias, and other bias also had a high risk of performance bias.

(b) Number of cases less than 200.

(c) Only one trial included, which was published in China and had a small sample size.

*The risk in the intervention group (and its $95 \%$ confidence interval) is based on the assumed risk in the comparison group and the relative effect of the intervention (and its 95\% CI). CI: confidence interval; MD: mean difference.

characteristics among those trials, it may point out that elder patients had poor self-administration ability and compliance to doctors' advice. Thus, health education may be more helpful for them to regularly use drugs, pay attention to the risk factors of diseases, and promote self-health behavior management.

In this review, the contents of HECM are composed by education of diet control, exercise, or other nonpharmaceutical therapy according to TCM theory and emotional balance. Since we did not compare the difference between HECM and other types of health education, we considered that partially or even large proportion of the effectiveness of HECM may be caused by the education itself. Recent research studies suggest that the improvements of SBP, FPG, BMI, smoking, and physical inactivity may reduce the premature mortality of NCDs [31]. However, despite joint efforts by public health and medical professionals in the last decade, awareness, treatment, and control of NCDs remain poor. Only $40.9 \%$ of hypertensive individuals aware of their condition and only $9.7 \%$ of them controlled their blood pressure well $(\mathrm{SBP} \leq 140 \mathrm{mmHg}$ and $\mathrm{DBP} \leq 90 \mathrm{mmHg}$ ) [32]. Therefore, health education is necessary for community patients with NCDs.

4.4. Implications for Clinical Practice and Future Research. According to the main findings and the quality of the evidence, the average blood pressure level of hypertension patients who accepted HECM was well controlled, and the average FPG level of diabetic patients was controlled as well (less than $7 \mathrm{mmol} / \mathrm{L}$ ). Thus, we suggest that community medical institutions should regularly carry out health education for patients with NCDs. The duration of the education should be at least 3 months, and the content of the education should contain medication guidance and general knowledge of disease prevention and treatment. The knowledge of health preservation and protection related to TCM can be educated according to patients' acceptance or operability.

The current very low quality of evidence is mainly due to the high risk of bias of the included studies; thus, we suggest researchers design and conduct high quality randomized controlled trials to testify the effectiveness of HECM in the future. To clarify the specific effectiveness of HECM, the control group could be non-TCM relevant health education. Sample size calculation should be done during the trial design period. Age of the patients could be considered as a stratification factor of randomization since we found the potential influence of age for the final outcomes. If the future study intends to compare the HECM and common type of health education, the outcome of health economics should also be included for cost-effectiveness analysis.

\section{Conclusion}

HECM, especially health education, seems to have advantages as an adjunctive therapy on improving the effectiveness of NCDs, including hypertension, diabetes, and CHD. However, due to the small sample size and potential bias of 
most included trials, this result should be interpreted with caution. More high-quality trials concerning long-term effects are warranted before the strong recommendation of HECM as a complementary therapy for NCDs.

\section{Additional Points}

Practical Implications. Community medical institutions could regularly carry out health education for patients with NCDs. The duration of the education should be at least 3 months, and the content of the education should contain medication guidance and general knowledge of disease prevention and treatment.

\section{Conflicts of Interest}

The authors declare that they have no conflicts of interest.

\section{Acknowledgments}

Hui-Juan Cao is supported by the National Natural Science Foundation of China (81804000) and Beijing Municipal Organization Department (2017000020124G292).

\section{References}

[1] World Health Organization, Noncommunicable DiseasesFactsheets, World Health Organization, Geneva, Switzerland, 2015.

[2] World Health Organization, Noncommunicable Diseases Country Profiles 2014, World Health Organization, Geneva, Switzerland, 2014.

[3] W. W. Chen, R. L. Gao, L. S. Liu, M. L. Zhu, and W. Wang, "China cardiovascular diseases report 2015: a summary," Journal of Geriatric Cardiology, vol. 14, pp. 1-10, 2017.

[4] Y. Xu, L. Wang, J. He et al., "Prevalence and control of diabetes in Chinese adults," JAMA, vol. 310, no. 9, pp. 948-949, 2013.

[5] A. Yang, H. M. Wu, J. L. Tang, L. Xu, and M. Yang, "Acupuncture for stroke rehabilitation," Cochrane Database Systematic Review, vol. 26, 2016.

[6] H. Abdi, M. Tayefi, S. R. Moallem et al., "Abdominal and auricularacupuncture reduces blood pressure in hypertensive patients," Complementary Therapies in Medicine, vol. 31, pp. 20-26, 2017.

[7] X. F. Zhao, H. T. Hu, J. S. Li et al., "Is acupuncture effective for hypertension? A systematic review and meta-analysis," PLoS One, vol. 10, no. 7, Article ID e127019, 2015.

[8] R. M. Nery, M. Zanini, J. B. de Lima, R. P. Bühler, A. D. da Silveira, and R. Stein, "Tai Chi Chuan improves functional capacity after myocardial infarction: a randomized clinical trial," American Heart Journal, vol. 169, no. 6, pp. 854-860, 2015.

[9] F. Stein, E. Boyle, E. Vayda, and R. H. Glazier, "A randomized controlled trial of a multifaceted integrated complementaryalternative therapy for chronic herpes zoster-related pain," Alternative Medicine Review: A Journal of Clinical Therapeutic, vol. 17, no. 17, pp. 57-68, 2012.

[10] C. S. Yi, K. Liao, J. Y. Yi, and X. F. Wang, “The clinical research of Preventive treatment of Chinese medicine on health service in community," Practical Clinical Journal of Integrated
Traditional Chinese and Western Medicine, vol. 16, pp. 41-43, 2016.

[11] Q. Liu, "Health education with characteristic of Chinese Traditional Medicine in community management role of type 2 diabetes," Chinese Journal of Nursing, vol. 18, pp. 641-643, 2012.

[12] Q. Shen, Q. H. Sun, F. L. Ni, and F. Y. Zhang, "The inqury analysis into the needs of the noncommunicable chronic disease sufferers by TCM rehabitation education," Chinese Journal of Clinical Healthcare, vol. 10, pp. 228-230, 2007.

[13] H. Y. Sun, "Health management in Chinese characteristics," International Journal of Nursing Sciences, vol. 36, pp. 649-651, 2017.

[14] J. H. Yang and Q. Wang, "Effect of diabetes health education with characteristic of Chinese traditional medicine on selfcare behavior in type 2 diabetics," Chinese General Practice, vol. 13, pp. 411-414, 2010.

[15] Y. Y. Ning, M. R. Zhong, Q. L. Deng, Y. X. Wang, and L. H. Wei, "The randomized controlled trial of Link healthcare of Chinese medicine on rehabilitation of post-percutaneous coronary intervention," Modern Journal of Integrated Traditional Chinese and Western Medicine, vol. 24, pp. 3281-3284, 2015.

[16] J. Higgins and S. Green, Cochrane Handbook for Systematic Reviews of Interventions, The Cochrane Collaboration, London, UK, 2nd edition, 2009.

[17] D. Atkins, D. Best, P. A. Briss et al., "Grading quality of evidence and strength of recommendations," BMJ, vol. 328, no. 7454, p. 1490, 2004.

[18] W. H. Ding, "The clinical research of healthcare and education of Chinese medicine on hypertension," Inner Mongol Journal of Traditional Chinese Medicine, vol. 34, pp. 178-179, 2015.

[19] X. P. He, "The effects of healthcare and treatment of Chinese medicine on hypertension in community," Chinese Medical Journal, vol. 47, pp. 254-255, 2015.

[20] L. Su, G. X. Liang, F. Q. Feng, and W. F. Chen, "The clinical research of health education of Chinese medicine on hypertensive patients in community," Journal of Contemporary Medicine, vol. 20, p. 69, 2014.

[21] P. Wu, "The clinical effects of health education of Chinese medicine on hypertensive patients in community," Modern Journal of Integrated Traditional Chinese and Western Medicine, vol. 22, pp. 928-930, 2014.

[22] H. L. Xie, Z. P. Wu, C. Liang, and L. F. Lan, "Application of health education in the management of 100 cases of hypertension in community," Chinese Journal of Ethnomedicine and Ethnopharmacy, vol. 25, pp. 124-125, 2016.

[23] L. M. Zhang, "The clinical research of health education of Chinese medicine on hypertensive patients in community," Clinical Journal of Traditional Chinese Medicine, vol. 25, pp. 340-342, 2013.

[24] Z. W. Hao and Z. J. Wang, "Observation on the effect of health education of traditional Chinese medicine in the prevention and control of diabetes mellitus," Guangming Journal of Chinese Medicine, vol. 34, pp. 635-638, 2019.

[25] L. Liu, W. Y. Mo, and W. J. Chen, "The clinical effects of health education of Chinese medicine on diabetes in community," Journal of Community Medicine, vol. 12, pp. 21-23, 2014.

[26] D. F. Shao and Z. F. Han, "Traditional Chinese medicine nursing and health education intervention in diabetic community," Chinese Baby, vol. 8, p. 264, 2018.

[27] F. X. Shao, "Evaluation of intervention effect of traditional Chinese medicine characteristic diabetes health education on community diabetes patients," Oriental Diet Therapy and Health Care, vol. 1, p. 269, 2018. 
[28] Z. Wu, "Application of health education for diabetes mellitus with Chinese characteristics in the management of type 2 diabetes mellitus," Diabetes New World, vol. 21, pp. 170-171, 2018.

[29] Y. Z. Yang, C. X. Wang, M. Luo, and Y. L. Xu, "The clinical research of healthcare of Chinese medicine on the quality of life of patients of angina pectoris," Chinese Medical Journal, vol. 47, pp. 291-293, 2015.

[30] X. D. Pu and P. Wu, "The effects of health education of Chinese medicine on improving the compliance of treatment in hypertensive patients," Journal of Frontiers of Medicine, vol. 3, pp. 17-18, 2013.

[31] Y. Li, X. Zeng, J. Liu et al., "Can China achieve a one-third reduction in premature mortality from non-communicable diseases by 2030?” BMC Medicine, vol. 15, no. 1, p. 132, 2017.

[32] Military Medical Science Press, National Center for Chronic and Non-Communicable Disease Control and Prevention, Chinese Center for Disease Control and Prevention. Report on Chronic Disease Risk Factor Surveillance in China, 2013, Military Medical Science Press, Beijing, China, 2016. 